\title{
Corela
}

Cognition, représentation, langage

HS-6 | 2007

Cognition, discours, contextes

\section{Comment formaliser en linguistique cognitive? Opération de fenêtrage et calcul du sens temporel}

\section{Gilles Col et Bernard Victorri}

\section{OpenEdition}

Journals

Édition électronique

URL : http://journals.openedition.org/corela/1504

DOI : $10.4000 /$ corela. 1504

ISSN : $1638-573 \mathrm{X}$

Éditeur

Cercle linguistique du Centre et de I'Ouest - CerLICO

Référence électronique

Gilles Col et Bernard Victorri, «Comment formaliser en linguistique cognitive ? Opération de fenêtrage et calcul du sens temporel », Corela [En ligne], HS-6 | 2007, mis en ligne le 01 novembre 2007, consulté le 21 avril 2019. URL : http://journals.openedition.org/corela/1504; DOI : 10.4000/corela.1504

Ce document a été généré automatiquement le 21 avril 2019

\section{(c) (i) (2)(2)}

Corela - cognition, représentation, langage est mis à disposition selon les termes de la licence Creative Commons Attribution - Pas d'Utilisation Commerciale - Partage dans les Mêmes Conditions 4.0 International. 


\title{
Comment formaliser en linguistique cognitive ? Opération de fenêtrage et calcul du sens temporel
}

\author{
Gilles Col et Bernard Victorri
}

1 Il s'agit dans cet article de réfléchir à la notion de formalisation en linguistique cognitive. L'émergence de la linguistique cognitive, et notamment de la grammaire cognitive de Langacker, est souvent présentée comme liée au rejet des approches formalisantes au profit du connexionisme présenté comme alternative (Langacker, 1991: 526). On ressent pourtant qu'il manque aux grammaires cognitives une formalisation forte, à un niveau d'intelligibilité plus adéquat pour l'analyse linguistique, qui leur permettrait, de surcroit, de jouer pleinement leur rôle dans les recherches pluridisciplinaires en sciences cognitives ( $c f$. Victorri 2004 : 98). La linguistique cognitive appartient en effet en partie à un domaine scientifique plus vaste que les sciences du langage, les sciences cognitives. C'est à ce double titre qu'il semble nécessaire qu'elle se dote d'un appareil formel cohérent et adapté à son objet d'étude. Or, peu de travaux ${ }^{1}$ ont été consacrés à ce jour à cet effort de formalisation. Il se trouve que parallèlement à la linguistique cognitive, il existe en France une école linguistique à tradition formelle et proche par bien des aspects de cette dernière. En effet si la Théorie des Opérations Prédicatives et Enonciatives (Culioli 1990, 1999a, 1999b) propose de considérer un énoncé comme le produit d'opérations de nature linguistique (prédication et énonciation), on peut facilement les décrire en termes cognitifs, en faisant ainsi apparaître clairement la proximité avec différents concepts de la linguistique cognitive. De plus, son objectif est de rechercher une large opérabilité de ses opérations (donc un certain degré de généralité). La théorie des repérages et le type de représentation topologique proposés dans ce cadre ont permis à une approche cognitive et "constructiviste " de se développer. Afin de réfléchir à l'ébauche d'une formalisation pour la linguistique cognitive, nous suggérons de mettre en regard la Théorie des Opérations Prédicatives et Enonciatives d'Antoine Culioli et la Grammaire Cognitive de Ronald Langacker, associée à la Sémantique Cognitive de Leonard Talmy. Leurs positions ne sont pas forcément toujours compatibles et leurs 
modèles de référence non plus mais ce qui rapproche ces deux théories, c'est le souci de chercher à décrire la construction et l'émergence du sens par une démarche noncompositionnelle, du moins au sens classique : on peut la qualifier de compositionnalité gestaltiste (cf. Victorri 1999).

C'est à travers l'exemple de l'opération de fenêtrage que nous présentons des éléments de formalisation en linguistique cognitive. Après une présentation de cette opération (section 1), nous prenons l'exemple de l'évocation de l'avenir dans une langue particulière (l'anglais) afin d'en montrer la pertinence (section 2). Enfin, nous introduisons dans la section 3 des éléments de calcul de l'opération de fenêtrage pour le traitement du sens prospectif à partir de l'exemple de l'anglais.

\section{L'opération de fenêtrage.}

3 La notion de «fenêtrage » est relativement classique et elle connaît une certaine actualité dans les travaux développés en sciences cognitives. Cette notion est liée à la notion de représentation, au sens étymologique de "rendre présent", qui déjà implique une dimension temporelle. Une représentation n'est effectivement pas donnée a priori, mais reconstruite. Ce processus de reconstruction s'accompagne du coup d'un décalage entre les événements et leur présentation dans le discours. Ce décalage donne alors à la représentation une dimension temporelle. La représentation est par ailleurs assimilable à un processus de déplacement d'attention. Comme le rappelle Gosselin (2005) qui définit le processus de représentation à partir des analyses du philosophe Husserl :

la véritable représentation [pour Husserl] consiste pour le sujet à déplacer son attention vers un autre moment du temps, disjoint du présent, et à se le représenter. (Gosselin $2005: 21$ )

La représentation peut ainsi se définir comme ayant à la fois une dimension temporelle et une dimension attentionnelle. Le décalage, ou la "dissociation» (Gosselin (2005)), introduite par la représentation se caractérise effectivement comme un écart entre deux moments, ce qui favorise l'émergence d'une dimension temporelle dans l'opération de fenêtrage. Le déplacement d'attention opéré par le sujet, qui se trouve ainsi à l'origine même du décalage, est quant à lui assimilable à l'ouverture d'une fenêtre sur un événement ou une partie d'un événement. Cette fenêtre contient alors une dimension temporelle dans la mesure où elle correspond à une re-présentation, au sens que l'on vient de définir. En tant que fenêtre, la représentation a une dimension spatiale (une fenêtre délimite, ou "partitionne" pour reprendre l'expression de Dinsmore (1991) l'espace dans lequel se déroulent des événements et dans lequel le sujet est impliqué2), ainsi qu'une dimension temporelle qui apparaît dans l'ordonnancement des événements dans la fenêtre. La notion de fenêtre, en tant que représentation envisagée comme portion d'espace-temps, est une notion essentielle dans les théories sémantiques cognitives actuelles. De manière plus générale, la théorie des "cadres " (Fillmore), des «espaces mentaux» (Fauconnier), des «espaces conceptuels» (Gärdenfors) ou, même plus proche de nous, des «domaines notionnels» (Culioli 1990, 1999a) ont au cœur de leur modèle une conception de la construction du sens en termes de monstration à travers une fenêtre, même si le terme en tant que tel n'apparaît essentiellement que chez un autre linguiste, Talmy (2000). Notre conception du «fenêtrage » est effectivement issue en grande partie de la notion de « windowing of attention » développée chez lui : 
Linguistic forms can direct the distribution of one's attention over a referent scene in a certain type of pattern, the placement of one or more windows of greatest attention over the scene, in a process that can be termed the windowing of attention. (Talmy, 2000, vol $1: 258$ )

Le «fenêtrage d'attention" concerne cependant chez Talmy l'inclusion ou l'omission d'éléments de la scène dans le discours. Notre conception tente plutôt d'intégrer la dimension temporelle dans la fenêtre d'attention, comme on le fait à travers la définition de la «scène verbale» (Victorri 1999). Le "fenêtrage» correspond finalement au processus de monstration de propriétés sémantico-cognitives en cours de structuration. Elle intervient directement au sein de la construction du sens. Dans cette optique, la temporalité a un double statut sémantique: c'est à la fois du sens construit par l'énonciation et en même temps un moyen de construire du sens, dans la mesure où la temporalité émerge du décalage créé par la représentation et le fenêtrage, et elle fait aussi partie des structures qui s'élaborent à travers la fenêtre. Les formes aspectotemporelles permettent d'évoquer autre chose que la temporalité : elles sont avant tout porteuses de sens. Une fenêtre est, par conséquent, nécessairement temporelle et, à ce titre, inscrit la construction du sens dans une certaine temporalité qu'il faut prendre en compte dans les analyses linguistiques.

Une présentation formelle et opératoire de l'opération de fenêtrage est présentée dans la dernière section de ce travail. De manière non formelle, comme on le voit dans la section qui suit, le fenêtrage correspond à la création et au repérage d'une fenêtre temporelle, ainsi qu'à la monstration d'une structure sémantico-cognitive. Du type de fenêtrage découle un certain type d'accès entre l'énoncé (et les unités linguistiques convoquées) et ce qu'il évoque, c'est-à-dire ce que les constructions linguistiques donnent à voir dans la fenêtre. La relation fenêtrage / accès pourrait se résumer par un principe régulateur d'« accroissement parallèle » : plus la structure est élaborée, plus elle est accessible. Afin de développer et d'expliciter cette définition, nous présentons certaines unités linguistiques de l'anglais qui permettent d'évoquer l'avenir et nous montrons comment ces unités contribuent à marquer l'opération de fenêtrage.

\section{Trois marqueurs linguistiques de l'opération de fenêtrage.}

7 Afin de présenter l'opération de fenêtrage, nous avons choisi de travailler sur les trois principales unités grammaticales qui permettent l'évocation de l'avenir en anglais, l'auxiliaire de modalité will, la périphrase be going to et la forme aspectuelle be+V-ing:

(1) A1G 32 'The situation is very, very fluid and there is a possibility that the regime will disintegrate' said one Western diplomat. (BNC : British National Corpus)

"La situation est très, très changeante et le régime pourrait s'effondrer" dit un diplomate occidental.

(2) BM5 1267 I'm not going to sit still and let them do that to me. (BNC) Je n'ai pas l'intention de rester assis tranquillement et de les laisser me faire ça.

(3) KCV 1052 I have my father on the phone half an hour later, erm, erm, when's Phil leaving tomorrow? (BNC)

J'ai mon père au téléphone une demi-heure plus tard, euh, euh... «Quand est-ce que Phil part demain?»

8 Ces trois unités ont comme point commun d'être des formes auxiliées, ce qui nous permet de montrer plusieurs aspects linguistiques et cognitifs de l'opération de fenêtrage dont 
elles sont des marqueurs. De notre point de vue, chaque élément linguistique contenu dans ces unités donne une indication d'une part sur la localisation de la fenêtre et d'autre part sur la caractérisation de la structure montrée dans la fenêtre.

Le principe général de l'opération s'inscrit dans une conception distributive de la construction du sens. Pour nous, la construction du sens se définit comme l'émergence de propriétés sémantiques globales non strictement réductibles à des unités discrètes. Nous avons ailleurs (Col sous presse) proposé l'hypothèse de la " prédication distribuée " qui donne à la construction du sens une étendue variable et une dynamicité indispensable. Dans ce contexte, l'opération de fenêtrage s'appuie sur deux familles de marqueurs, l'auxiliaire et le verbe ${ }^{3}$ :

- l'auxiliaire, ou le semi auxiliaire dans le cas de be going to, indique la position de la fenêtre, c'est-à-dire, il indique si la fenêtre est connectée ou déconnectée de la situation de référence (la situation d'énonciation-origine).

- le verbe, sous la forme non finie (sous la forme de la base verbale dans le cas de will, de l'infinitif en to dans le cas de be going to et enfin affecté du morphème -ing dans le cas de be $+V$-ing) indique ce qui est montré dans la fenêtre. Les différentes formes verbales non finies marquent différents degrés de détermination de l'occurrence de procès: la base verbale correspond à une occurrence indéterminée, la forme en To + base verbale correspond à une occurrence brute et quantitative, la forme V-ing correspond à une occurrence déterminée et qualitative. Ces trois degrés de détermination permettent, sur le plan sémantique et cognitif, de caractériser la structure montrée dans la fenêtre et d'en préciser le degré d'élaboration.

Le fenêtrage permet ainsi de rendre compte de l'évocation du sens temporel de l'énoncé. Ce sens est construit à la fois par la fenêtre (le «cadre » lui-même) et sa position, ainsi que par son contenu, c'est-à-dire la structure sémantique montrée dans la fenêtre. L'évocation temporelle revient pour nous à la localisation d'une fenêtre et la caractérisation de ce qui est montré dans la fenêtre. Le point de vue cognitif adopté dans ce travail n'est donc pas un point de vue métaphorique et spatial comme c'est le cas dans les grammaires cognitives nord-américaines, mais un point de vue qui fait du temps une simulation linguistique avant tout, essentiellement basée sur l'opération de fenêtrage.

11 Examinons maintenant, du point de vue du fenêtrage, les unités qui permettent l'évocation temporelle.

\section{Be going to $+\mathrm{V}$}

Les analyses menées sur be going to dans le cadre de la Théorie des Opérations Prédicatives et Énonciatives (parmi d'autres, Celle 1997, ou Col et Duchet 2000) montrent que cette forme présente la réalisation de l'événement à venir dans la continuité de la situation de référence. On relève que les différents moments, moment d'énonciation et moment de validation du procès envisagé, sont certes différents, mais situés sur le même plan, donc connectés l'un à l'autre. Pour être plus précis, on a ancrage d'une occurrence du procès go to dans la situation d'énonciation, et cet ancrage est représenté dans l'énoncé grâce à be qui marque l'opération d'identification. Quant à l'occurrence du procès $\mathrm{V}$ (dans [going to V]) représenté par la base verbale, elle est repérée par rapport à un moment $t$ différent du moment qui sert de référence $\left(\mathrm{t}_{0}\right)$. Ce moment est malgré tout connecté à $\mathrm{t}_{0}$ : il est sur le même plan d'énonciation que lui. Ce marqueur n'indique pas l'opération de "visée » au sens défini par Culioli ${ }^{4}$ : on est dans une problématique où le choix entre $p$ et $p$ ' est fait, donc le chemin vers la validation est à la fois tracé et continu. Cette présentation dans 
l'optique culiolienne permet de rendre compte de la valeur donnée à cette forme dans la tradition anglo-saxonne. Palmer ([1979] 1990 : 144) par exemple, parle de « futur du point de vue du présent (future from the standpoint of the present) et de «current orientation ». Be going to se distingue nettement de will par exemple qui, lui, marque l'opération de "visée » (voir plus loin). Cette différence de moments permet d'envisager que la fenêtre temporelle marquée par cette forme grammaticale est connectée elle aussi à la situation de référence.

La forme verbale non finie (то + base verbale) ${ }^{5}$ indique de son côté que la structure sémantique évoquée est peu déterminée. Cette forme correspond à une occurrence de procès extraite du domaine notionnel associé. Elle marque que l'on a affaire à une occurrence fragmentée et quantitative de la notion. Une notion est un système de représentation de différentes propriétés physico-culturelles associé à un ensemble d'unités linguistiques. Par exemple, la notion /drink/ en anglais se manifeste par «a drink», " to drink», «drinking», " glass ", «bottle», " pub», etc. quelle que soit la catégorie grammaticale à laquelle ces unités appartiennent. En outre, une notion se représente topologiquement par un espace dynamique appelé "domaine», qui se divise en sous-domaines. Ces sous-domaines sont représentés linguistiquement par des " occurrences ", c'est-à-dire des unités nominales ou verbales. Pour être complet, ces sous-domaines sont spécifiés et déterminés par des opérations marquées par des unités grammaticales. Une occurrence de notion, qu'elle soit verbale ou nominale, est ainsi le produit d'un ensemble d'opérations de détermination. Dire que l'on a affaire avec то + base verbale à une occurrence fragmentée et quantitative de la notion revient à dire que l'on a en quelque sorte " une structure brute » dont on distingue seulement les contours, dans la mesure où elle correspond à une détermination quantitative. Par extension, on considère que le marqueur To convoque dans ce cas une structure brute dont il indique l'accessibilité (voir là aussi Col 2001). Nous utilisons ici la notion d'accès (et d'accessibilité) dans le sens où un énoncé donne à voir du sens à travers des structures sémantico-cognitives qui s'élaborent à partir des unités linguistiques présentes dans l'énoncé, ainsi qu'à partir de leurs relations ${ }^{6}$. Dans le cadre d'une théorie de l'évocation de l'avenir, une structure "élaborée » est parallèlement une structure dont l'accès est ouvert. C'est cet accroissement parallèle élaboration / accessibilité qui est central dans la construction du sens prospectif. Une prédiction «certaine» (si on adopte temporairement un point de vue épistémique) correspond en termes cognitifs et formels à la monstration dans une fenêtre temporelle d'une structure bénéficiant d'un certain degré d'élaboration et d'accès. La notion de certitude n'est en fait guère pertinente, et on peut lui substituer les notions d'accessibilité et d'éboration: le sens prospectif qui émerge de l'énoncé est plus ou moins élaboré et accessible, et l'événement prédit est plus ou moins visible dans la fenêtre temporelle. La spécificité du sens prospectif réside en fait, et de manière générale pour nos trois marqueurs, dans la sous-élaboration des structures, et donc dans leur manque d'accès. C'est ce même rôle de marqueur d'accessibilité qu'il faut voir en To dans be going to. Ce rôle est en fait plus visible quand on compare la forme « pleine » avec la forme réduite et orale gonna, comme dans l'exemple suivant :

(4) A6Y 977 Oxford United fans, for example, were found to have a repertoire of over 250 carefully orchestrated chants ranging from 'We're gonna win the league, tra la la la' to 'You're gonna get your fuckin' heads kicked in...,' the favoured greeting for visiting fans. (BNC)

On a constaté que les supporters de Oxford United par exemple ont un répertoire de plus de 250 chants soigneusement orchestrés, allant de "On va gagner la ligue, tra la 
la la “ à “On va vous défoncer la tête...", l'accueil réservé aux supporters des équipes invitées. deux formes du point de vue du fenétrage. Si on adopte pour la démonstration un point de vue spatial, on constate que ce qui est montré dans la fenêtre avec be going to, c'est le point d'arrivée - lui-même représenté par la forme non finie то + base verbale - du mouvement métaphorique exprimé par la périphrase entière, c'est-à-dire la validation dont l'accès est tracé. A l'inverse, ce qui est montré avec gonna, c'est une structure plus indéterminée dans la mesure où le point d'arrivée du mouvement métaphorique n'est pas montré et, en conséquence, la validation non plus. To est effectivement assimilé au verbe par un phénomène de réduction accentuelle et de centralisation vocalique : on passe de [going to V] à [gonna V] $]^{7}$. L'hypothèse défendue dans Col et Duchet est alors que la validation est plutôt suggérée que montrée. Si on prend comme critère les adverbes temporels par exemple, on observe qu'ils sont peu nombreux dans le co-texte de gonna si on compare avec celui de be going to, et qu'une bonne partie d'entre eux a plusieurs emplois possibles. Parmi eux, on relève surtout des adverbes employés dans un sens modal (notamment just), ce qui contribue à montrer dans la fenêtre non pas l'événement à venir, mais plutôt les conditions et l'origine de la prédiction. Dans l'extrait suivant par exemple (issu d'un corpus oral),

(5) A: [No] you don't have to be horrible you just have to go up and say...

B: Oh God <unclear>

A: You're not gonna be made a fool of.

B: But I can't make that point cos it just gets enemies,

A: Well so you're just gonna carry on being nice and get loads of friends.

Non, tu n'as pas à être méchant. Tu as seulement à te lever et dire...

Oh mon Dieu

On va pas se moquer de toi

Mais je peux pas faire ça, on se fait des ennemis

Bon, t'as quà continuer d'être gentil et avoir plein d'amis.

(COLT, Corpus of London Teenagers)

l'adverbe just contribue à fenêtrer non pas l'événement initialement souhaité mais un autre qui est présenté en quelque sorte "par défaut ». On est d'une certaine façon dans une logique davantage modale que spatiale.

En résumé, la forme be going to marque qu'une structure dont on ne voit que les contours est montrée dans une fenêtre connectée à la situation de référence. Les deux formes que l'on présente dans les paragraphes suivants s'opposent à be going to, tant sur le plan de la position de la fenêtre que sur celui de l'élaboration de la structure montrée dans la fenêtre.

\section{Be+V-ing vs. will}

La forme aspectuelle be+V-ing et le modal will illustrent deux types de fenêtrage très différents l'un de l'autre. Will est un auxiliaire de modalité et à ce titre, il marque un calcul sur la validation de la relation prédicative. Be+V-ing , en tant que forme aspectuelle, renvoie plutôt à la problématique du point de vue sur le déroulement du procès. Les deux marqueurs s'opposent fondamentalement du point de vue des repérages énonciatifs, dans la mesure où be+V-ing marque l'identification du moment du procès à venir $t_{i}$ avec le moment d'énonciation $t_{0}^{8}$, tandis que will marque la rupture entre ces moments. La 
conséquence de cette opposition est que la fenêtre temporelle qu'ils contribuent à construire se trouve soit connectée à la situation d'énonciation et identifiée à elle (be+Ving), soit déconnectée et du coup inaccessible dans le cas de will. Ce qui permet de définir la fenêtre, son contenu en quelque sorte, est également différent d'un marqueur à un autre. Will se construisant avec la base verbale, ce qui est donné à voir dans la fenêtre est relativement indéterminé. La fenêtre elle-même est en quelque sorte "pauvre" puisqu'aucune structure précise n'est montrée. Le point de vue énonciatif est marqué ailleurs, au niveau de l'auxiliaire, et il est d'ordre modal. Cette analyse en termes de fenêtre et de structure correspond à ce que l'on peut observer à propos de ce modal quand il sert à construire le sens temporel. Il est effectivement très dépendant du cotexte pour sa valeur prospective: "Will est une forme repérée et nécessite une forte détermination pour exprimer la projection» (Celle 1997: 41). La valeur prospective repose effectivement sur un repérage de type quantitatif et non qualitatif comme le font les autres valeurs de will. En tant que modal, la problématique que pose will relève en fait davantage de la question « comment créer un accès ? " que de la question «à quoi accèdet-on?» (ce qu'expriment plutôt les deux autres formes principales de construction du sens prospectif, be+V-ing et be going to, même si elles posent aussi, mais dans une moindre mesure, la question : « comment créer un accès ? »). Prenons les deux exemples suivants :

(6) Once she understands the consequences of her actions, she'll go easy on you.

Une fois qu'elle comprendra les conséquences de ses actions, elle ne sera pas trop dure avec toi.

(7) Travis: If we're following the wrong car, we're gonna' have to wait a whole other month.

Hunter : - I'll be eight then.

Travis : Si on suit la mauvaise voiture, va falloir qu'on attende à nouveau un mois entier.

Hunter : J'aurai huit ans à ce moment-là.

(script du film Paris Texas : 77)

Ils montrent explicitement que la visée exprimée par will dépend soit d'une condition et d'une autre prédiction comme en (7), soit de la validation d'un premier procès comme en (6). C'est en ce sens que la structure sémantico-cognitive construite par l'énoncé avec will n'est pas vraiment montrée dans la fenêtre temporelle; on pourrait dire qu'elle n'est qu'au stade d'amorce vu que sa dépendance par rapport à une condition ou une prédication préalable est établie. Il se dessine le principe suivant: plus la structure est élaborée plus elle est accessible. Dans le cas de will et de la forme verbale associée (base verbale), l'accès est justement quasi-nul dans la mesure où la structure est sous-élaborée, la base verbale marquant une forme de sous-détermination. On constate ainsi, d'un point de vue méthodologique, que les opérations énonciatives de détermination de la notion trouvent très naturellement leur place dans une approche cognitive de l'analyse linguistique et lui apportent déjà des éléments de formalisation parfaitement opératoires.

De son côté, be+V-ing indique à la fois que la fenêtre est connectée à la situation d'énonciation par identification avec celle-ci (voire confondue comme en (8) et (9) plus bas, en l'absence de circonstants temporels qui permettraient de la localiser), et que la structure est suffisamment élaborée pour qu'une partie seulement soit montrée dans la fenêtre. La monstration d'une partie de la structure correspond au rôle de marqueur d'inaccompli de be+V-ing. Ni la fin ni le début du procès ne sont montrés dans la fenêtre : c'est l'«intérieur» de la structure qui est montré dans la fenêtre. Le concept de «fenêtre » temporelle est, de fait, proche de celui d'aspect. La différence fondamentale 
entre le fenêtrage et la représentation aspectuelle par intervalle réside néanmoins dans le contenu de l'espace de représentation. Une fenêtre n'est pas seulement un cadre défini par des dimensions et une position. Il n'y a de fenêre sans contenu; une fenêtre se construit avec et par son contenu. Inversement, le contenu construit la fenêtre. Ainsi, la structure sémantico-cognitive n'est pas indépendante de la fenêtre, elle n'a pas de statut d'objet dont des parties seulement seraient présentes dans le cadre de la fenêtre. Le fenêtrage implique ainsi une double élaboration : celle du « cadre » lui-même, et celle de la structure avec le cadre. On remarque encore une fois que le modèle cognitif développé ici s'appuie sur des opérations formelles et topologiques, et s'inscrivent dans la suite de l'idée culiolienne d'«Intérieur du domaine notionnel» (Culioli, 1990:122) autant que celle de « perspective interne » (internal perspective) présente chez Langacker (1991: 209). La monstration d'une partie de la structure en élaboration est par ailleurs compatible avec d'autres éléments de sens évoqués par le marqueur, comme par exemple l'intention en (4), construite à l'aide de la négation autant que par be+V-ing, ou encore la planification en (5) :

(8) Emmy was not going to Will's concert. She had decided not to even think about it. (A. Lurie, Love and Friendship : 207)

Emmy n'avait pas l'intention d'aller au concert de Will. Elle avait décidé de ne même pas y penser.

(9) A: We're both leaving. We've got it all planned.

B: I'm leaving. I'm getting out of here. [...] I'm clearin' outa' here once and for all.

A : On s'en va tous les deux. On a tout prévu.

$\mathrm{B}:$ Je pars. Je me barre. Je me tire de là une bonne fois pour toutes.

(S. Shepard, True West : 53)

L'émergence des idées d'intention et de planification contribuent à faire de be+V-ing un marqueur d'anticipation davantage que de prédiction, au sens où l'événement, ou du moins une partie de l'événement, est envisagé et, pour ainsi dire, "amené » dans la situation d'énonciation repère. Dans ce type de co-texte sans repère prospectif explicite, l'anticipation vient de la nature des procès et du point de vue aspectuel. Leave, get et clear out dans nos exemples sont lexicalement des procès à bornes confondues, donc à intervalle aspectuel nul. Malgré tout, be+V-ing, en tant que marqueur de fenêtrage, tend à mettre en perspective ces procès et à faire qu'une partie de la structure soit montrée dans la fenêtre. Un déroulement purement simulé est créé, dont une partie est donnée à voir sur la scène verbale. Le rôle anticipatoire que joue be+V-ing explique aussi l'absence de nécessité d'un repère prospectif dans l'énoncé. Quand il apparaît :

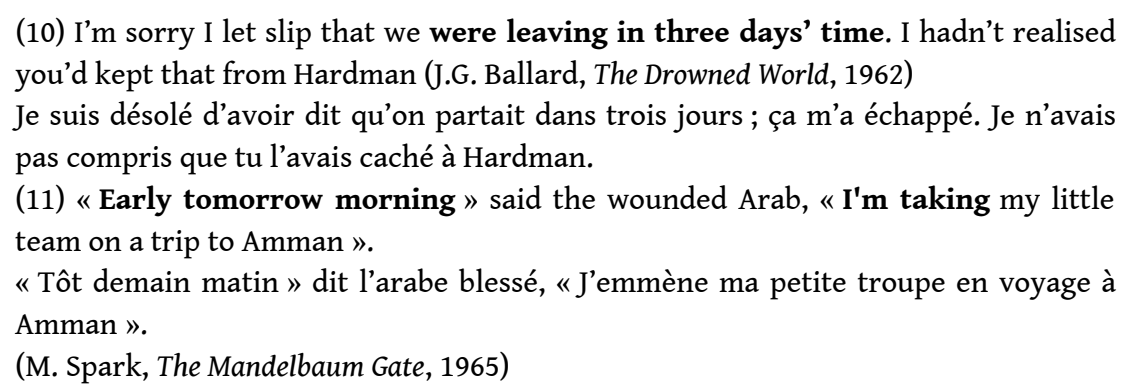

21 il prend sa part dans le fenêtrage. Dans (11) par exemple, " early tomorrow morning " contribue explicitement à la position de la fenêtre et par conséquent à la position de ce qui est montré. Même si le moment repère est prospectif, le repérage de la fenêtre marqué par la forme be+V-ing n'est pas modifié: on a toujours identification entre le moment repère et le moment du procès qui, dans ce type d'énoncé, est explicite. Ce qu'apporte le repère prospectif, et sa position initiale et focale dans l'énoncé va dans ce 
sens, c'est plutôt une spécification - au sens de "précision » - sur l'état d'élaboration de la structure, et donc une spécification sur l'accessibilité à la structure émergeant de l'énoncé. Pour ce qui est de "in three days' time» en (10), on remarque que la détermination supplémentaire apportée par le repère prospectif quantifie l'écart entre le moment repère de l'anticipation et le moment repère prospectif, ce qui contribue à quantifier l'accès à la structure et à lui donner une "granularité » plus élevée, pour reprendre une notion utilisée en intelligence artificielle.

\section{Bilan.}

La monstration d'une partie de la structure sémantico-cognitive correspondant à un énoncé évoquant l'avenir contribue ainsi à faire de la construction du sens prospectif (voire plus largement du sens temporel) une problématique d'accès. «Accès » doit être compris du point de vue cognitif, dans le sens où la fenêtre donne à voir une représentation sous forme de structure plus ou moins élaborée. Les trois marqueurs principaux observés tendent effectivement à faire de la structure sémantico-cognitive une structure dont on ne voit pas toutes les dimensions. La base verbale seule, TO + base verbale, ou encore V-ing, représentent en définitive trois cas d'accès limité, soit parce que la structure est brute et sous-déterminée (base verbale), soit parce qu'on ne voit que les contours de la structure sans plus (то + base verbale), soit enfin parce qu'on ne voit qu'une partie de la structure et le reste n'est pas montré dans la fenêtre (V-ing). Dans la section suivante, nous proposons des éléments de calcul de l'accès, ainsi que de la position, du contenu et des dimensions de la fenêtre ; il semble pour l'instant essentiel d'insister sur le fait que cet accès limité à la structure constitue la spécificité cognitive de l'évocation du futur en anglais.

$\mathrm{Si}$ on résume les observations précédentes, l'évocation de l'avenir en anglais se caractérise pour nous par la création d'une fenêtre (une représentation envisagée comme une portion d'espace-temps, $c f$. section 1), par le repérage de cette fenêtre par rapport à la situation d'énonciation (qui donne une valeur à la fenêtre) et par la monstration dans cette fenêtre de l'élaboration d'une structure sémantico-cognitive. La forme grammaticale de temps (présent ou prétérit) et le type d'auxiliaire indiquent spécifiquement le repérage de la fenêtre par rapport à la situation d'énonciation d'origine. La forme du verbe, forme non finie dans nos trois marqueurs, indique l'état d'élaboration de la structure. Elle ne caractérise pas la structure en tant que telle : celle-ci est évoquée par l'ensemble de la construction linguistique (la forme verbale complexe et les autres unités linguistiques, comme les circonstants de temps par exemple) en même temps que la fenêtre. Rappelons que ce que nous appelons " fenêtrage ", c'est l'ensemble de l'opération - repérage de la fenêtre + monstration de la structure - , et que du type de fenêtrage découle un certain type d'accès entre l'énoncé et ce qu'il évoque, suivant le principe régulateur d'« accroissement parallèle » : plus la structure est élaborée, plus elle est accessible. On retrouve dans cette amorce de modèle (au risque de simplification) les valeurs généralement attribuées aux trois marqueurs pris en compte :

- "planification» et "anticipation" pour be+V-ing qui donne à voir l'intérieur d'une structure, correspondant ainsi à un état d'élaboration relativement important ;

- "intention» dans le cas d'un sujet animé ou «connaissance d'un fait extérieur " (Celle 1997: 43) pour be going to qui donne à voir les contours d'une structure peu élaborée (l'événement brut en quelque sorte); 
- « volonté » ou « simple futur » de will avec la condition que le repère futur soit posé dans le co-texte dans la mesure où justement l'accès à la structure est un enjeu de l'énonciation (rôle modal de will).

\section{Eléments de formalisation de l'opération de fenêtrage dans l'évocation de l'avenir.}

Nous proposons dans cette dernière partie quelques éléments de formalisation de l'opération de fenêtrage. Nous rappelons que l'hypothèse défendue ici est que les formes linguistiques de l'anglais « actives » dans l'évocation de l'avenir se décomposent en deux unités qui marquent d'une part la position de la fenêtre temporelle (auxiliaire be ou will) et d'autre part le contenu et les limites de la fenêtre (forme verbale non finie). Cette hypothèse cognitive repose sur les opérations linguistiques de repérage temporel d'une part, et de détermination aspectuelle d'autre part (structuration aspectuel de l'intervalle de déroulement du procès). Pour poser quelques éléments de formalisation et, afin de proposer une forme de calcul des fenêtres temporelles, nous nous servons d'ensembles mathématiques et de relations entre ensembles. Notre point de vue formel se distingue ainsi de celui de Talmy qui n'envisage pas de calcul à proprement parler et pour qui le fenêtrage dépend de la présence ou non de certains éléments linguistiques :

The most fundamental formal linguistic device that mediates this cognitive process is the inclusion in a sentence of explicit material referring to the portion or portions of the total scene that are to be foregrounded, and the omission of material that would refer to the remainder of the scene intended for backgrounding. (Talmy $2000: 258$ )

L'intérêt de la formalisation que nous proposons est de considérer le sens temporel d'un point de vue non spatial ou métaphorique, comme c'est le cas actuellement dans le courant américain de la linguistique cognitive (chez Talmy ou Langacker). Nous souhaitons plutôt montrer que le sens temporel est le résultat d'une construction dynamique. Si la linguistique cognitive s'appuie sur des processus cognitifs réguliers, il semble cependant indispensable de formaliser ces processus afin de pouvoir redéfinir dans une approche cognitive non spatialiste le rôle des unités linguistiques dans l'énoncé.

\section{Principe général.}

Soit $\mathrm{E} 1$, un ensemble de départ contenant un sujet de départ $(\mathrm{A})^{9}$, un repère temporel de départ (B) et un procès de départ (C).

Soit $\mathrm{E} 2$, un ensemble d'arrivée correspondant à l'événement à venir et contenant un sujet envisagé ( $\left.A^{\prime}\right)$, un repère temporel à venir $\left(B^{\prime}\right)$ et un procès envisagé $\left(C^{\prime}\right)$.

Soit $\mathrm{R}$ un ensemble de relations asymétriques entre les éléments de E1 et de E2, orientées de E1 vers E2. On dira qu'une relation de $\mathrm{R}$ est satisfaite si l'élément de E1 sert de repère effectif à l'élément de E2 correspondant, i.e. si la relation entre ces deux éléments s'appuie sur une relation de repérage à valeur d'identification ou de différenciation (au sens de Culioli, 1990 : 137-138); en revanche, la relation n'est pas satisfaite s'il y a rupture (toujours au sens culiolien) entre les deux éléments correspondants. 
Soit $\mathrm{F}$ un cadre pouvant contenir des relations entre les éléments de $\mathrm{E} 1$ et E2, donc une partie de R ou R entier. En les contenant, $\mathrm{F}$ montre ces relations, et masque celles qui ne sont pas dans le cadre.

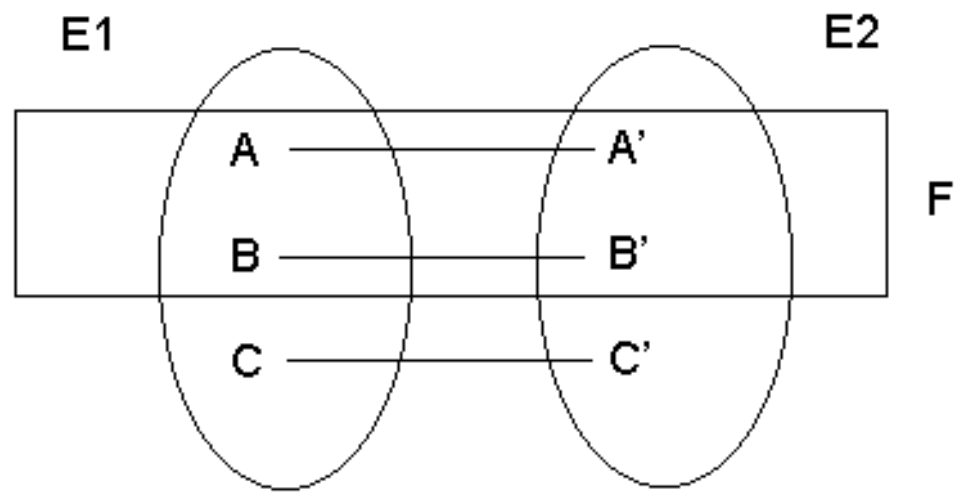

\section{Figure 1: Formalisation du fenêtrage (cas d'un énoncé contenant be+Ving)}

A partir de ces outils de formalisation par ensembles, on peut envisager plusieurs cas de figure de relations entres les éléments de E1 et E2 et par extension plusieurs cas de fenêtrage et d'accès à la structure cognitive évoquée, en fonction de chacune des formes linguistiques en jeux.

$1^{\text {er }}$ cas :

Les relations entre E1 et E2 sont toutes satisfaites. On obtient deux types possibles de fenêtrage :

- Soit F contient et montre toutes ces relations satisfaites

- Soit F ne contient et ne montre qu'une partie de ces relations

Le premier type de fenêtrage ne semble néanmoins pas pouvoir correspondre au sens prospectif dans la mesure où la spécificité de ce sens est justement que la structure montrée dans la fenêtre est sous-élaborée. En revanche, le second type de fenêtrage correspond à la forme be+V-ing évoquant l'avenir. L'auxiliaire be indique que toutes les relations sont satisfaites dans la mesure où il marque fondamentalement que les éléments de $\mathrm{E} 1$ et $\mathrm{E} 2$ sont dans une relation d'identification. La forme $V$-ing marque quant à elle que toutes les relations ne sont pas montrées, le morphème -ing marquant l'inaccompli d'un point de vue aspectuel. La nature de B' (repère associé au procès envisagé), en l'occurrence prospective, permet également de spécifier le contenu de la fenêtre (voir plus bas à propos de la relation $\left.\left(\mathrm{B}, \mathrm{B}^{\prime}\right)\right)$.

\section{$2^{\mathrm{e}}$ cas :}

Les relations entre E1 et E2 ne sont pas toutes satisfaites et $\mathrm{F}$ montre toutes les relations satisfaites et seulement elles.

Be going to marque ce second cas de relations entre E1 et E2. Les relations ne sont pas toutes satisfaites en raison de la présence de deux procès différents contenus dans E1 (go to) et E2 (V). Seules les relations satisfaites sont montrées, compte tenu de la détermination représentée par la forme verbale : (to) base verbale ${ }^{10}$. 
$3^{\mathrm{e}}$ cas : certaine façon, la spécification de l'une contribue à la spécification de l'autre selon le principe régulateur d'«accroissement parallèle » mentionné dans la seconde partie. Par ailleurs, la fenêtre étant par définition "pleine », elle montre quelque chose, même si ce quelque chose peut hypothétiquement se ramener à une valeur nulle. Fenêtrer revient à montrer quelque chose rendu accessible parce que contenu dans la fenêtre. Ainsi, les trois formes verbales concernées (correspondant à la construction [auxiliaire + forme non finie]) contribuent à la mise en place de la fenêtre et de l'accès que la fenêtre construit en se mettant en place. Dans la problématique de l'évocation de l'avenir, l'accès est justement un enjeu : il n'y a pas de véritable accès à la structure cognitive.

ses dimensions. Ces caractéristiques sont construites :

- par le repérage de E2 par rapport à E1. Ce repérage est marqué linguistiquement par l'auxiliaire d'une part et la nature du repère temporel associé au procès (B') d'autre part. Ce repérage construit la position de la fenêtre.

- par le type d'accès entre les ensembles, qui élabore le contenu de la fenêtre (ce qu'elle montre, $c$ f. plus bas) et qui lui donne une partie de ses dimensions (limites du cadre). Le contenu et les dimensions sont marqués par la forme du verbe lui-même (forme non finie) ainsi que par l'opération de détermination à laquelle elle renvoie (fléchage, extraction, ou encore « renvoi à la notion »).

- par le type d'intervalle aspectuel évoqué par la forme linguistique globale, qui donne d'autres dimensions à la fenêtre.

\section{d'établir}

(11) «Early tomorrow morning » said the wounded Arab, «I'm taking my little team on a trip to Amman ».

«Tôt demain matin » dit l'arabe blessé, «J'emmène ma petite troupe en voyage à

Amman ».

(M. Spark, The Mandelbaum Gate, 1965)

41

En (11), les deux repères $B$ et $B^{\prime}$ de chacun des ensembles, le moment de référence et le moment associé à la validation, se situent dans une relation d'identification marquée par 
l'auxiliaire be. La relation (B, B') est ainsi satisfaite. Pour ce qui est de (C, C'), la marque aspectuelle d'inaccompli fait que le procès take est anticipé : on le voit par avance - d'où la relation satisfaite $\left(\mathrm{A}, \mathrm{A}^{\prime}\right)$ également. Plus précisément, on n'en voit qu'une partie parce que son intervalle est ouvert. C'est par conséquent $\left(C, C^{\prime}\right)$ qui ne peut être entièrement montrée, d'où son « masquage » par la fenêtre. On a affaire au cas présenté dans la figure 1 plus haut dans lequel $\left(C, C^{\prime}\right)$ n'appartient pas à $F$ bien que cette relation soit satisfaite. La fenêtre temporelle correspondante contient alors une partie de l'intervalle aspectuel du procès de E2 (la borne de départ), ainsi que les relations (A, A') et (B, B').

Pour ce qui est de (12) :

(12) After an interval the man spoke to me. He said that my friend was a very rough boy and asked did he get whipped often at school. I was going to reply indignantly that we were not National School boys to be whipped, as he called it; but I remained silent. (J. Joyce, Dubliners)

Au bout d'un moment, l'homme me parla. Il dit que son ami était un garçon brutal, et demanda si on lui donnait souvent le fouet à l'école. Je faillis répliquer avec indignation que nous n'étions pas à l'école publique et qu'on ne nous donnait pas le fouet pour reprendre son expression ; mais je ne dis rien.

Dublinois, J. Joyce. Trad. J. Aubert, Gallimard, coll. Folio.

Les repères temporels de chacun des procès (go et reply) sont construits comme différents dans la mesure où going to $V$ établit une distinction entre eux (on n'a pas d'identification comme avec be+Ving en (11)). La relation (B, B'), bien que contenant des éléments différents (valeur de différenciation du repérage), est satisfaite malgré tout : le repère de go to est simplement différent de celui de reply mais il n'a pas rien à voir avec lui. C'est encore $C$ et C' qui ne sont pas montrés dans F. Plus exactement, et si on reprend la différence entre be going to et gonna, seul C' (le procès de E2) est montré mais pas la relation $\left(C, C^{\prime}\right)$ qui n'est pas satisfaite. L'exemple (12) où est énoncée une intention qui en définitive ne mène à rien ( $I$ remained silent ») va dans le sens de cette absence de relation (C, C'). La fenêtre contient seulement les deux relations (A, A') et (B, B') :

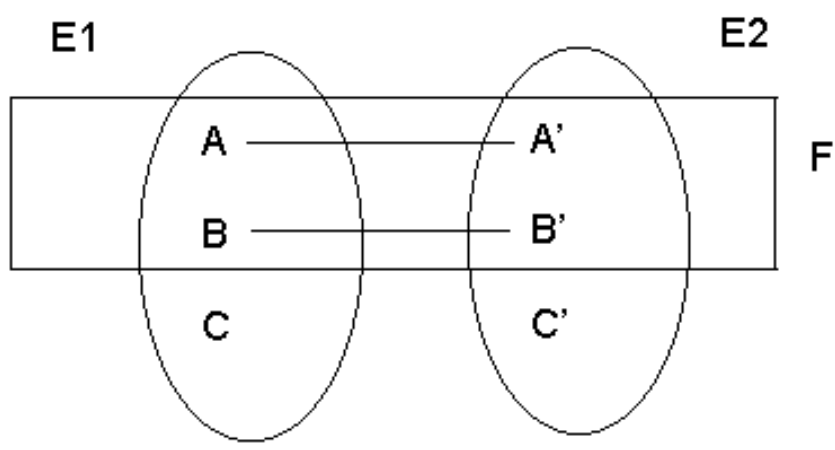

Figure 2: Formalisation du fenêtrage correspondant à un énoncé contenant be going to

Le cas du modal will est différent des deux autres dans la mesure où la valeur de rupture du repérage temporel propre aux modaux fait que la relation B et B' n'est pas satisfaite. La forme du procès visé, la base verbale (c'est-à-dire une forme indéterminée qui représente l'ensemble du domaine notionnel marqué par le verbe), fait que la relation ( $C$, $\left.C^{\prime}\right)$ n'est pas satisfaite non plus. La fenêtre ne contient ainsi que (A, A') et ne montre ainsi que la relation subjective, ce qui correspond aux valeurs particulières de l'évocation temporelle avec will, comme la «bonne volonté » ou la "caractéristique du sujet» (cf. 
Palmer ([1979] 1990) pour une description approfondie des modaux de l'anglais, ainsi que Gilbert (2001) pour une présentation de will dans le cadre de la TOPE) :

(13) Little Chandler smiled, looked confusedly at his glass and bit his lower lip with three childishly white front teeth.

"I hope you'll spend an evening with us," he said, "before you go back. My wife will be delighted to meet you. We can have a little music and..."

"Thanks awfully, old chap," said Ignatius Gallaher, "I'm sorry we didn't meet earlier. But I must leave tomorrow night." (J. Joyce, Dubliners)

Little Chandler sourit, regarda son verre d'un air confus, et trois incisives, blanches comme celles d'un enfant, mordirent sa lèvre inférieure.

- J'espère que tu passeras une soirée chez nous, dit-il, avant ton départ. Ma femme sera ravie de faire ta connaissance. Nous pourrons faire un peu de musique et...

- Merci infiniment, mon vieux, dit Ignatius Gallaher. Je regrette qu'on ne se soit pas rencontrés plus tôt. Mais je dois partir demain soir.

Dublinois, J. Joyce. Trad. J. Aubert, Gallimard, coll. Folio.

Avec le premier will (sous la forme 'll), le personnage de Little Chandler fait appel à la bonne volonté de Gallaher, ce qui met en place une relation entre sujets qui détermine l'évocation temporelle; la relation (A, A') est ainsi satisfaite, et donc montrée dans la fenêtre. Le second will renvoie quant à lui indirectement à cette bonne volonté, dans le sens où la validation du second procès dépend de la validation du premier : «she will be delighted if you spend an evening with us ». La relation (A, A') est également satisfaite. La relation $\left(A, A^{\prime}\right)$ est également évidente en (14), mais pour des raisons légèrement différentes :

(14) "Who knows?" he said, as they lifted their glasses. "When you come next year I may have the pleasure of wishing long life and happiness to Mr. and Mrs. Ignatius Gallaher."

Ignatius Gallaher in the act of drinking closed one eye expressively over the rim of his glass. When he had drunk he smacked his lips decisively, set down his glass and said:

"No blooming fear of that, my boy. I'm going to have my fling first and see a bit of life and the world before I put my head in the sack ... if I ever do."

"Some day you will," said Little Chandler calmly. Ignatius Gallaher turned his orange tie and slate-blue eyes fullupon his friend.

"You think so?" he said.

"You'll put your head in the sack," repeated Little Chandler stoutly, "like everyone else if you can find the girl." (J. Joyce, Dubliners)

- Qui sait? dit-il alors qu'ils levaient leurs verres. Quand tu viendras l'an prochain, j'aurais peut-être plaisir de souhaiter longue vie et bonheur à Mr et Mrs Ignatius Gallaher.

Ignatius Gallaher, qui était en train de boire, eut un clin d'œil expressif par dessus le bord de son verre. Lorsqu'il eut fini, il fit claquer ses lèvres d'un air péremptoire, posa son verre et dit:

- Mon vieux, tu n'as fichtre pas à t'en faire pour ça. Je vais commencer par m'en payer une bonne tranche, faire un peu la vie, circuler un peu, avant de me mettre la corde au cou - si ça doit jamais arriver.

- Ca arrivera bien un jour, dit Little Chandler calmement.

Ignatius Gallaher braqua sa cravate orange et ses yeux bleu ardoise sur son ami.

- Tu crois ça ? dit-il.

- Tu te mettras la corde au cou, répéta résolument Little Chandler, comme tout le monde si tu trouves la fille.

Dublinois, J. Joyce. Trad. J. Aubert, Gallimard, coll. Folio.

On voit à travers cet exemple que l'évocation temporelle passe ici par les propriétés du sujet, en l'occurrence ce qui apparait dans cet extrait comme une fatalité chez l'homme 
de se marier : "you'll put your head in the sack ». On remarque même que le passage de "I'm going to have my fling first... before I put my head... » à « someday you will » s'effectue par un recadrage sur (A, A') au détriment de (B, B') dans la mesure où B' se trouve indéterminé (someday). Le passage de be going to à will va dans le sens de ce qui se passe en général avec cet auxiliaire : la fenêtre ne montre plus que (A, A') qui se trouve être la seule relation satisfaite entre E1 et E2 (cf. Figure 3) :

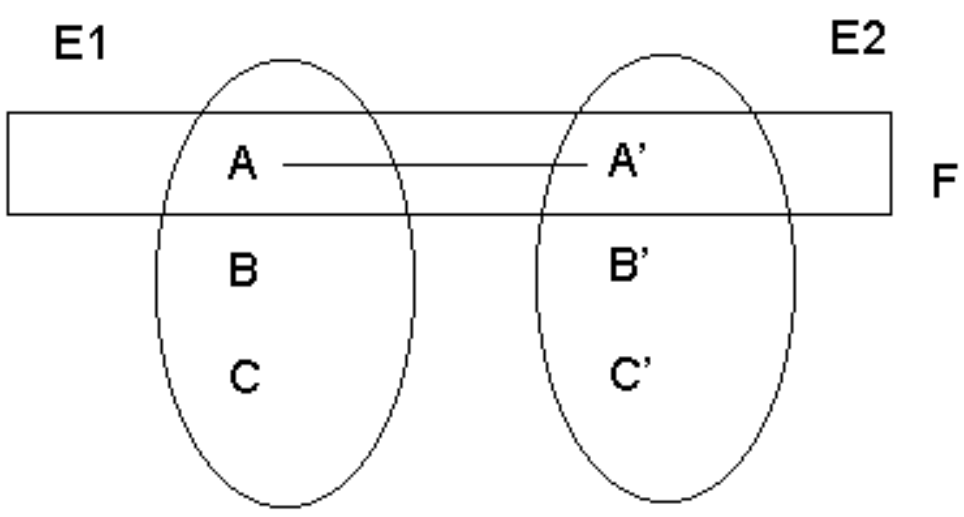

\section{Figure 3 : Formalisation du fenêtrage d'un énoncé contenant will}

L'énoncé (10) et le passage de be going to à will accompagné du masquage de la relation (B, B') pourraient en fait se décrire comme un cas de « refenêtrage ».

\section{L'accès à la structure cognitive montrée dans la fenêtre.}

On vient de voir que la fenêtre temporelle contient des relations satisfaites entre des éléments de E1 et E2. D'un point de vue formel, ces relations composent la structure cognitive correspondant au sens temporel évoqué par l'énoncé et en donnent le degré d'élaboration; ce dernier dépend en définitive de $\mathrm{R}$ ( $\mathrm{R}$ correspond à l'ensemble des relations entre les éléments de E1 et ceux de E2). La structure cognitive est ainsi accessible en fonction des relations entre ensembles: on accède à la structure par les relations satisfaites entre E1 et E2. Formellement, on peut envisager que l'accès se calcule à partir d'elles. On peut alors distinguer, à partir des différences de contenu et de taille de fenêtres observées pour nos trois formes $b e+V$-ing, be going to et will, trois types d'accès à la structure cognitive en fonction du nombre de relations satisfaites et montrées dans $F$. Les relations entre ensembles sont dites « satisfaites » en fonction :

du type de repérage du procès par rapport à la situation repère. On obtient ainsi trois cas de figure :

- $\mathrm{B}$ ', le repère temporel du procès, et $\mathrm{B}$, le repère de l'ensemble de départ, sont identifiés. Toutes les relations toutes satisfaites, mais elles ne sont pas nécessairement toutes montrées (cf. Figure 1).

- $B$ ', le repère temporel du procès et $B$, le repère de l'ensemble de départ, sont différenciés. Seules les relations (A, A') et (B, B') sont satisfaites. 
- $B$ ', le repère temporel du procès, et $B$, le repère de l'ensemble de départ, sont déconnectés. Seule la relation (A, A') est satisfaite.

des éléments de E2 (en l'occurrence C') correspondant à la forme verbale et au type de détermination de la notion de procès qu'elle marque ${ }^{11}$ :

- si l'occurrence de procès est fléchée, alors C' est repris d'un autre ensemble (cas de be+Ving)

- si l'occurrence de procès est extraite, alors C' est introduit dans E2 (cas de be going to)

- si l'occurrence de procès est visée, C' n'est pas stabilisé et ne peut satisfaire la relation (C, C') (cas de will)

52 La relation $\left(\mathrm{A}, \mathrm{A}^{\prime}\right)$ quant à elle semble toujours satisfaite dans la mesure où, soit le sujet est agentif et la relation est en quelque sorte «motivée » par cette agentivité (qui prend la forme de l'intention, ou de la (bonne) volonté), soit il est non agentif et, en ce cas, des exemples comme les suivants montrent que le locuteur (l'origine du texte) prend plus spécifiquement en charge la relation et établit $\left(\mathrm{A}, \mathrm{A}^{\prime}\right)$ :

(15) CH8 517 The laugh she gave was so dirty that I knew it was going to be all right. (BNC)

Le rire qu'elle eut était si grossier que je sus que ça allait bien se passer.

En (15), la prédiction est rendue certaine par «I knew » d'un côté et la locution so + adj. + that qui marque une conséquence suite à l'établissement d'un haut degré de l'adjectif dirty et renforce la visée. (A, A') est satisfaite. Dans le cas d'un sujet impersonnel avec will, comme en (16):

(16) ED3 1618 The machine has a large, touch-sensitive, digital screen that displays clear instructions --; and, should you make a mistake in a procedure, it will show you the correct step, so you really can't go wrong. (BNC)

La machine a un grand écran digital sensible au toucher et qui affiche des instructions claires-; et en cas d'erreur de procédure, il vous montrera les étapes correctes, donc vous ne pouvez pas vous tromper.

les propriétés du sujet ne sont pas prises directement en considération pour permettre d'avoir une relation satisfaite entre $\left(\mathrm{A}, \mathrm{A}^{\prime}\right)$. It renvoie effectivement à " the machine ». En revanche, le co-texte fournit des éléments qui permettent de satisfaire la relation. D'une part l'énoncé qui précède expose toutes les propriétés de l'écran (notamment «clear instructions »), ce qui contribue à asserter la visée exprimée par will; d'autre part l'énoncé qui suit, "so you really can't go wrong", renforce lui aussi la visée par son caractère déductif (so), ainsi que par le rejet de la solution "go wrong » au profit de la solution alternative (dans une perspective de bifurcation ; cf. Culioli, 1990 : 113).

Un dernier exemple montre enfin comment la fenêtre temporelle permet non seulement de représenter l'évocation temporelle correspondant à une forme grammaticale, mais aussi l'évolution de la fenêtre, sur le modèle du « refenêtrage » vu précédemment :

(17) "O, Mr. Conroy, will you come for an excursion to the Aran Isles this summer? We're going to stay there a whole month. It will be splendid out in the Atlantic. You ought to come. Mr. Clancy is coming, and Mr. Kilkelly and Kathleen Kearney. It would be splendid for Gretta too if she'd come. She's from Connacht, isn't she?"

"Her people are," said Gabriel shortly.

"But you will come, won't you?" said Miss Ivors, laying her arm hand eagerly on his arm.

"The fact is," said Gabriel, "I have just arranged to go..." (J. Joyce, Dubliners)

- Oh, dites, Mr Conroy, voulez-vous venir en excursion aux îles d'Aran cet été? Nous allons y rester un mois entier. Ce sera merveilleux d'être en plein Atlantique. Vous devriez venir. Il y aura Mr Clancy, et Mr Killkelly et Kathleen Kearney. Ce 
serait merveilleux pour Gretta aussi, si elle venait. Elle est bien originaire du Connacht?

- Sa famille, oui, répondit Gabriel brièvement.

- Mais vous viendrez, vous, n'est-ce pas? dit-elle en posant avec ardeur une main chaude sur son bras.

- A vrai dire, fit Gabriel, j'ai déjà pris mes dispositions pour aller...

Dublinois, J. Joyce. Trad. J. Aubert, Gallimard, coll. Folio.

« it will » permet de recentrer la monstration uniquement sur (A, $\left.A^{\prime}\right)$, c'est-à-dire non pas seulement sur l'assertion d'un événement programmé ("going to stay »), mais sur les caractéristiques de l'événement («it will be splendid»). La suite du paragraphe fait que d'autres personnages sont intégrés dans la fenêtre, qui en quelque sorte s'élargit : «Mr. Clancy is coming, and Mr. Kilkelly and Kathleen Kearney ». Be+V-ing permet ainsi de montrer une autre facette de l'événement à venir (et donc de la structure cognitive émergeant de l'énoncé) qui n'est pas montrée au début du passage. Au cours de ce refenêtrage, la relation (C, $C^{\prime}$ ) est alors satisfaite, mais elle n'était toujours pas contenue dans la fenêtre (on est toujours dans le cas de la Figure 1 où (C, $C^{\prime}$ ) est en dehors de F). On voit par ailleurs que c'est au niveau discursif que le caractère évolutif et dynamique de la fenêtre apparaît nettement.

\section{Limites du modèle.}

Cette première ébauche de formalisation de l'opération de fenêtrage est loin d'être complète. Nous nous sommes tout d'abord concentrés sur les formes verbales complexes, ainsi que sur l'évocation de l'avenir. Il va sans dire que le modèle doit être complété par d'autres formes et pour d'autres évocations temporelles. L'objectif à long terme est d'arriver à proposer une formalisation de la construction du sens (à partir de l'exemple de l'anglais) qui prenne en compte le rôle de la temporalité, à la fois bien sûr comme sens construit, mais aussi comme élément constructeur de sens.

Dans le même ordre d'idée, notre formalisation repose sur des énoncés dans lesquels les circonstants temporels sont simples et coordonnés. Pour être opératoire, la formalisation doit intégrer des cas plus complexes, par exemple les cas de subordination dans des exemples comme :

(18) G0L 1785 Your men will not be told the location until they arrive there. (BNC)

Le lieu ne sera pas connu de vos hommes jusqu'à ce qu'ils arrivent.

On sera alors amené à proposer d'autres éléments de formalisation, davantage dynamiques et qui prendront en compte des relations entre plusieurs ensembles dans la mesure où des relations complexes entre moments se mettent en place.

De manière plus technique, deux questions essentielles devront trouver une réponse.

61 La première concerne la relation $\left(C, C^{\prime}\right)$ établie entre les procès. Cette relation joue un rôle important dans la formalisation dans la mesure où elle permet d'introduire l'idée que même si une relation entre deux éléments de chacun des ensembles est satisfaite, elle n'est pas nécessairement montrée dans $\mathrm{F}$. La condition de la satisfaction n'est donc pas une condition nécessaire. La relation (C, C') permet également de distinguer deux cas proches du point de vue du fenêtrage : celui de be+V-ing et celui de be going to ( $c f$. les figures 1 et 2). Dans les deux cas, $F$ montre les relations satisfaites (A, A') et (B, B'), mais la relation ( $\left.C, C^{\prime}\right)$ est soit satisfaite mais non contenue dans $F(b e+V-i n g)$, soit non satisfaite ( 
be going to). Il semble cependant que la relation (C, $\left.C^{\prime}\right)$ ne soit pas de même nature que les deux autres. Elle est d'une certaine façon déséquilibrée : c'est surtout l'élément $C$ ' qui joue un rôle essentiel dans la formalisation, notamment dans l'accès à la structure contenue dans la fenêtre. Qui plus est, $\mathrm{C}$ lui-même ne renvoie pas vraiment à un procès dans le cas de be+V-ing et celui de will, et dans le cas de be going to, c'est le verbe go qui est vraiment concerné. Nous devons donc mettre en évidence une formalisation qui permette de remplir les fonctions de (C, C') et de contribuer à la distinction entre le fenêtrage propre à $b e+V$-ing et à celui de be going to. Cette formalisation devra aussi permettre de définir l'accès à la structure cognitive à défaut (éventuellement) d'une relation (C, $\left.C^{\prime}\right)$.

62 La seconde question concerne les conflits de repérage qui peuvent apparaître entre l'aspect propre au verbe (exemple: aspect statif) et l'aspect véhiculé par la forme grammaticale (exemple : aspect inaccompli). Ce type de conflit de repérage est en soi non problématique et des éléments de résolution de conflit seront mis en évidence. Le plus important est pour nous d'intégrer les conflits dans la formalisation, ce qui implique d'en augmenter la dynamicité et d'introduire la notion de repérage par défaut.

\section{Conclusion.}

En réponse à la question que nous posons dans le titre de cet article, nous avons tenté de définir des éléments de formalisation dans le domaine de la linguistique cognitive. Nous sommes partis de l'exemple de l'opération de fenêtrage, opération qui permet de rendre compte d'un point de vue énonciatif et surtout cognitif, de la construction du sens temporel. Nous proposons alors une formalisation qui s'inspire de la théorie des ensembles, avec comme objectif la mise en place d'éléments de calcul du sens temporel, en l'occurrence de calcul de la position, du contenu et des dimensions de la fenêtre temporelle.

64 Cette formalisation n'en est bien entendu qu'au stade d'ébauche pour le moment et de plus, elle s'appuie sur un nombre limité d'unités linguistiques. Elle tend cependant à montrer qu'il est possible, voire nécessaire, de formaliser en linguistique cognitive. A partir du moment où on met en évidence des opérations, qu'elles soient énonciatives ou cognitives, il est indispensable de proposer des éléments formels visant à montrer leur caractère vraiment opératoire.

Pour rendre encore davantage opératoire le fenêtrage, il est maintenant indispensable d'étendre les observations et les analyses à d'autres formes aspecto-temporelles ou à d'autres unités comme par exemple certaines prépositions dans leurs emplois temporel ( until, by, etc.) et les conjonctions de temps ${ }^{12}$, ainsi qu'à d'autres langues. L'appareil formel dont on présente une esquisse ici devrait également s'enrichir, ce qui renforcera son caractère opératoire. 


\section{BIBLIOGRAPHIE}

Bryant, J. et Mok, E 2002. « Constructing English Conditionals: Building Mental Spaces in ECG », Univ. of California, http://www.icsi.berkeley.edu/ jbryant/BryantMok290.pdf

Celle, A. 1997. Etude contrastive du futur français et de ses réalisations en anglais. Numéro spécial de la collection Linguistique Contrastive et Traduction. Ophrys.

Col, G. 2001. « Infinitif en TO et accès à une scène verbale ». Anglophonia n¹0. Toulouse : Presses Universitaires du Mirail. 109-123.

Col, G. 2007. Eléments de modélisation énonciative et cognitive de la construction du sens. Habilitation à Diriger des Recherches, Document de synthèse. Université de Poitiers.

Col, G. 2007 (sous presse). « Windowing the future. The cognitive operation of 'windowing' in the study of future time evocation », in Du fait grammatical au fait cognitif / From Gram to Mind: Grammar as Cognition, J.-R. Lapaire, et al. (eds), 12 pages. Presses Universitaires de Bordeaux.

Col, G. Sous presse. « "Relation " ou “ intégration ” prédicative ? La prédication comme principe d'émergence du sens ", in La Prédication, Bibliothèque des Faits de Langues, sous la responsabilité de J.-M. Merle. Gap, Ophrys. 10 pages.

Col, G. et Duchet, J.-L. 2000. « Éléments pour une définition des valeur de gonna en anglais, à partir du corpus électronique COLT », in Cahiers FORELL, n¹4. Poitiers, Maison des Sciences de l'Homme et de la Société. 167-186.

Culioli, A. 1990. Pour une linguistique de l'énonciation, vol. 1. Ophrys

Culioli, A. 1999a. Pour une linguistique de l'énonciation. vol. 2. et 3. Ophrys.

Culioli, A. 1999b. « Accès et obstacle dans l'ajustement intersubjectif ». In Pour une linguistique de l'énonciation, vol. 3. 91-99. Ophrys.

Dinsmore, J. 1991. Partitioned Representations. Dordrecht, Boston, London : Kluwers Academic Publishers.

Fauconnier, G. 1997. Mappings in Thought and Language. Cambridge, CUP.

Fillmore, C. 1982. «Frame Semantics ». In Linguistics in the Morning Calm, Linguistic Society of Korea (ed.). Seoul, Hanshin, 111-138.

Gärdenfors, P. 2000. Conceptual Spaces. The Geometry of Thought. MIT Press.

Gilbert, E. 2001. « Vers une analyse unitaire des modalités », Cahiers de Recherche en Grammaire Anglaise, 8. Ophrys. 23-99.

Gosselin, L. 2005. Temporalité et modalité. De la représentation comme dispositif sémantique. Duculot.

Langacker, R. 1991. Foundations of Cognitive Grammar. Vol. II : Theoretical Prerequisites. Stanford , Cal. : Stanford University Press.

Luzzati, D. et Mir-Samii, R. 1996. « La transition de l'accord par le relatif : vers une théorie du fenêtrage ». Faits de langues n8. Ophrys. 193-202.

Mok, E., Bryant J. et Feldman J.A. 2004. « Scaling Understanding up to Mental Spaces », Proceedings of ScaNaLU-2004, Boston, MA, http://acl.ldc.upenn.edu/W/W04/W04-2806.pdf 
Palmer, F. R. [1979] 1980. Modality and the English Modals. London and New York : Longman.

Talmy, L. 2000. Toward a Cognitive Semantics. Volume 1. London, Cambridge, The MIT Press.

Tomasello, M. 1999. The Cultural Origins of Human Cognition. Cambridge, Mass., London, England : Harvard University Press.

Victorri, B. 1999. « Le sens grammatical », Langages 136. 85-105.

Victorri, B. 2004. « Les grammaires cognitives », in La Linguistique cognitive, Fuchs, C. (dir.). Gap :

Ophrys, et Paris : Maison des Sciences de l'Homme. 73-98.

\section{NOTES}

1. On peut citer notamment les travaux de l'équipe de Jerry Feldman à Berkeley : cf. entre autres Bryant et Mok (2002) et Mok, Bryant et Feldman (2004).

2. Voir également les travaux de Tomasello sur le Partitioning Process (1999: 150): «the process of taking different perspectives on events as the speaker windows and gaps different aspects of the event for the listener» (Tomasello 1999: 156). Par ailleurs, certains travaux portant sur le traitement de la syntaxe et de la prosodie, notamment Luzzati et Mir-Samii (1996), utilisent la notion de fenêtrage.

3. Le choix de prendre en compte ces deux familles de marqueurs est un choix méthodologique. Des analyses menées actuellement au laboratoire FORELL permettent d'intégrer d'autres unités dans l'opération de fenêtrage, comme les prépositions until et by.

Les remarques qui suivent reprennent de manière synthétique celles développées dans Col 2007 (sous presse).

4. «Le futur implique une visée. On entend par là que, du repère énonciatif $S_{i t}$, on vise une

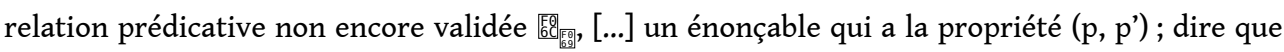

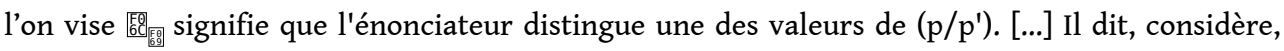
espère, veut, ordonne, craint, suppute, etc. que, en $T_{i}$ [repère à-venir et "moment de validation »], la relation prédicative sera validée. [...]. Ainsi en $T_{1}$, [repère de locution] $=T_{0}$ [repère de l'énonciation], on $a\left(p, p^{\prime}\right)$ et l'on vise $p$ en $T_{i}$. [...] Il y a rupture entre l'énonciation de la visée $\left(\mathrm{T}_{1}=\mathrm{T}_{\mathrm{o}}\right)$ et sa validation par le biais de l'énoncé $\left(\mathrm{T}_{\mathrm{i}}\right.$, moment de validation) ». (Culioli, 1990 : 146-147).

5. Le statut et le rôle de to posent des problèmes en soi qui ne sont pas traités ici. Pour une discussion sur ces questions d'un point de vue cognitif, voir Col 2001.

6. La notion d'accès est présente dans plusieurs théories énonciatives et cognitives, de Culioli pour qui « toute mise en relation [de deux termes] (x R y) équivaut à la construction d'un chemin d'accès entre $\mathrm{x}$ et $\mathrm{y}$ (eux-mêmes construits) (1999: 91) à Fauconnier qui en fait un principe s'inspirant de la projection d'un ensemble sur un autre et de la relation entre éléments de chaque ensemble : «If two elements $a$ and $b$ are linked by a connector $F(b=F(a))$, then element $b$ can be identified by naming, describing or pointing to its counterpart a ». (Fauconnier, $1997: 41$ )

7. La réduction accentuelle et la centralisation vocalique qui l'accompagne sont, de manière générale, dans la phonologie de l'anglais, un facteur facilitant pour des phénomènes d'assimilation, de palatalisation en présence de [j] et de compression syllabique. Dans le cas de gonna, il se produit un phénomène d'assimilation de la nasale vélaire [C] dans ['gFxqC] par le trait apico-alvéolaire $\mathrm{du}[\mathrm{t}]$ qui le suit pour aboutir à ['gFxqntF]. Vient à sa suite un abrègement par smoothing de la séquence diphtongue + voyelle en une diphtongue [cq] d'où ['gcqntF] et enfin de réduction de la diphtongue à la voyelle brève ['gontF].

8. Les deux moments $t_{i}$ et $t_{0}$ sont en fait les termes d'une relation de repérage dans laquelle le moment du procès à venir $t_{i}$ est repéré par rapport au moment d'énonciation $t_{0}$ (terme repère). 
Cette relation de repérage peut prendre plusieurs valeurs : identification, différentiation et rupture.

9. Les termes "sujet de départ ", " repère temporel de départ » et " procès de départ " doivent être compris dans le sens où la projection de E1 sur E2 est orientée de E1 vers E2.

10. Comme on l'a vu à propos de going to vs. gonna, l'appartenance de to à la forme verbale non finie est en soi une problématique, d'où sa mise entre parenthèses dans la notation.

11. Les termes de fléchage, d'extraction et de visée sont utilisés ici aussi dans le sens de la théorie culiolienne. En un mot, l'opération de fléchage consiste à évoquer une instance déjà construite et l'opération d'extraction à en construire une à partir d'un domaine notionnel, alors que l'opération de visée, comme nous l'avons vu (cf. note 4), laisse en suspens la construction d'une nouvelle instance.

12. Ce travail est actuellement en cours dans le cadre de l'opération «PréPost » du laboratoire FORELL (EA3816, MSHS Poitiers).

\section{RÉSUMÉS}

Ce travail s'inscrit dans un projet plus vaste visant à élaborer une formalisation forte dans le domaine de la linguistique cognitive. Notre modèle s'appuie sur la Théorie des Opérations Prédicatives et Énonciatives qui considère un énoncé comme le produit d'opérations de nature linguistique, opérations que l'on peut également décrire en termes cognitifs. La théorie des repérages et le type de représentation topologique proposé dans ce cadre permettent à la fois une approche cognitive et constructiviste. Nous présentons dans cette perspective l'opération de fenêtrage qui contribue à une analyse cognitive des marques aspecto-temporelles de l'anglais. Cette opération permet de replacer la problématique aspecto-temporelle dans une perspective d'accès à une structure sémantico-cognitive émergente. Ces éléments de formalisation, qui se veulent fondamentalement non algébriques et non compositionnels, pourraient par ailleurs donner à la linguistique un rôle conséquent dans les recherches pluridisciplinaires en sciences cognitives.

This work is part of a broader project aiming at developping a thorough formalization in the field of cognitive linguistics. Our model is based upon the Theory of the Predicative and Enunciative Operations (TOPE). TOPE considers an utterance as the product of various linguistic operations which can easily be described in cognitive terms. The theory of location and the type of topological representation suggested in the TOPE framework enables us to propose both a cognitive and constructional approach. From this angle, we present the operation of windowing which contributes to a cognitive analysis of English aspectual and temporal markers. The operation of windowing enables to reconsider Time and Aspect as a question of access to an emerging semantic, cognitive structure. These elements of formalization, claiming non-algebraic, non-compositional roots, may give linguistics a consistent role in pluridisciplinary research in cognitive science. 
INDEX

Mots-clés : accès, construction dynamique du sens, élaboration, fenêtrage, fenêtre temporelle, formalisation, prédication distribuée, repérage, structure sémantico-cognitive, temps / aspect / modalité, théorie des ensembles, Théorie des Opérations Enonciatives et Prédicatives (TOPE), sémantique cognitive

Keywords : access, cognitive semantics, distributed predication, dynamic construction of meaning, formalization, location, semantic, cognitive structure, set theory, tense / aspect / modality, Theory of the Predicative and Enunciative Operations (TOPE), time window, windowing

\section{AUTEURS}

\section{GILLES COL}

Université François-Rabelais, Tours / Laboratoire FORELL-EA 3816, Poitiers

\section{BERNARD VICTORRI}

Laboratoire LATTICE (UMR 8094), CNRS / Ecole Normale Supérieure et Université Paris-Diderot 